\title{
SU(2) gauge theory with domain-wall fermions in fundamental and adjoint representations
}

\author{
Hideo Matsufuru* \\ High Energy Accelerator Research Organization (KEK), Tsukuba 305-0801, Japan, \\ and Graduate University for Advanced Studies (Sokendai), Tsukuba 305-0801, Japan \\ E-mail: hideo.matsufurudkek.jp
}

\section{Kei-ichi Nagai}

Kobayashi-Maskawa Institute for the Origin of Particles and the Universe (KMI), Nagoya University, Nagoya, 464-8602, Japan

E-mail: keiichi.nagaidkmi.nagoya-u.ac.jp

\section{Norikazu Yamada}

High Energy Accelerator Research Organization (KEK), Tsukuba 305-0801, Japan, and Graduate University for Advanced Studies (Sokendai), Tsukuba 305-0801, Japan E-mail: norikazu.yamadadkek.jp

\begin{abstract}
We numerically study the SU(2) gauge theories with fermions in fundamental and adjoint representations. For the fundamental fermions, we perform dynamical simulations with $N_{f}=2,4,6$, and 8 using the domain-wall fermions to examine the $N_{f}$ dependence of the meson masses and decay constants. For the adjoint fermions, we investigate, for advance preparation for simulations at a fixed topological charge, the properties of the vacuum in the presence of two-flavors of Wilson fermions with and without the twisted mass ghosts. The meson spectrum is calculated to clarify the Aoki phase structure.
\end{abstract}

The 33rd International Symposium on Lattice Field Theory

14 - 18 July 2015

Kobe International Conference Center, Kobe, Japan

\footnotetext{
* Speaker.
} 


\section{Introduction}

In seeking for a candidate of walking technicolor [U, ㅁ] ], which may resolve not only conceptual drawbacks including the fine-tuning problem but also practical deficit in the standard model such as absence of dark matters, the phase structures of gauge theories in variety of gauge groups, fermion representations, and the number of flavors have been nonperturbatively studied in lattice simulations [3]. Among these theories, from the viewpoint of chiral symmetry breaking, the SU(2) gauge theory is unique as the breaking pattern is different from QCD. Because of the pseudoreality of the $\mathrm{SU}(2)$ group, for fermions in the fundamental representation, the breaking pattern is $S U\left(2 N_{f}\right) \rightarrow S p\left(2 N_{f}\right)$, while for $S U\left(N_{c}\right)$ group with $N_{c} \geq 3$ it is $S U\left(N_{f}\right) \times S U\left(N_{f}\right) \rightarrow S U\left(N_{f}\right)$. For the adjoint fermions, the symmetry breaking is realized as $S U\left(2 N_{f}\right) \rightarrow S O\left(2 N_{f}\right)$ for any number of colors, $N_{c}$. The study of gauge theories with adjoint fermions is also important to understand the structure of the $\mathscr{N}=1$ super Yang-Mills theory.

In this work, we study the $\mathrm{SU}(2)$ lattice gauge theories with fermions in fundamental and adjoint representations. For the fundamental fermions, we adopt the domain-wall fermion action and perform dynamical simulations with $N_{f}=2,4,6$, and 8 . The results obtained so far have been reported in Refs. [四, 回]. On these ensembles, in this paper, we measure the decay constant of the pseudoscalar meson to see their $N_{f}$ dependence.

As for the adjoint fermions, we are preparing to apply chirally symmetric fermions such as the domain-wall or the overlap operators, where absence of locality or large simulation costs can be a source of difficulty. Both potential problems are related to the near-zero modes of the Hermitian Wilson Dirac operator in the kernel, $\gamma_{5} D_{W}$, and could be worse as the lattice size increases. In general, exploring the conformal window requires large volume simulations. Then, the introduction of the topology fixing term is useful to have better locality and suppress the simulation cost [ [6, 四]. However, the topology fixing term has to be incorporated with care, because the lower end of the conformal window for the SU(2) gauge theory with adjoint fermions is expected to be at around $N_{f}=2$ and hence putting the topology fixing term, which made of two flavors of Wilson fermions and ghosts, could drastically change the dynamics or even the critical number of flavors. Thus, we investigate the Aoki phase structure in the quenched approximation before proceeding to dynamical simulations.

In the next section, we report the results for the SU(2) gauge theory with dynamical domainwall fermions in the fundamental representation. Section 3 shows the study of Aoki phase in the presence of the topology fixing term as well as the Wilson fermions (corresponding to the topology fixing term without the twisted mass ghosts). Section $⿴$ is devoted to our conclusions and outlook.

\section{Fundamental Representation with Domain-wall Action}

Numerical simulations are performed on $16^{3} \times 32$ lattices with the Iwasaki gauge action and domain-wall fermions with $N_{f}=2,4,6$, and 8 [ [4, 5]. The simulations are performed with the extent of 5-th direction $L_{s}=16$ and the domain-wall height $M_{0}=1.6$. Table $\mathbb{0}$ summarizes the parameters of generated ensembles as well as the lattice scale $r_{0}$. In Ref. [四] we reported the results of the static potential and the meson spectrum. These studies show that the result for $N_{f}$ larger than 6 is qualitatively different from those with $N_{f}<6$. In particular at $N_{f}=8$ the feature of the 


\begin{tabular}{ccccc}
\hline & & \multicolumn{3}{c}{$m$} \\
$N_{f}$ & $\beta$ & 0.20 & 0.10 & 0.05 \\
\hline 2 & 0.85 & $2.3419(77)$ & $2.623(14)$ & $2.827(22)$ \\
& 0.90 & $2.874(15)$ & $3.194(15)$ & $3.472(20)$ \\
\hline 4 & 0.85 & $2.842(21)$ & $3.551(43)$ & $4.215(89)$ \\
& 0.90 & $3.528(26)$ & $4.702(51)$ & $5.849(74)$ \\
\hline 6 & 0.80 & $2.462(13)$ & $3.403(32)$ & $4.851(83)$ \\
& 0.85 & $3.396(20)$ & $5.172(67)$ & $8.42(35)$ \\
& 0.90 & $4.611(46)$ & $6.551(90)$ & $10.61(52)$ \\
\hline 8 & 0.80 & $2.988(36)$ & $4.667(62)$ & $8.33(25)$ \\
& 0.85 & $4.317(44)$ & $7.15(16)$ & $15.9(1.1)$ \\
\hline
\end{tabular}

Table 1: Parameters of numerical simulations of the SU(2) gauge theory with fundamental fermions and the value of $r_{0}$ in each ensemble.

confinement and chiral symmetry breaking tends to disappear as fermion mass vanishes. In this work, to reinforce these results, we measure the decay constant of the pseudoscalar meson.

We apply the standard method to extract the decay constant from the local-to-local pseudoscalar correlators. Figure $\mathbb{W}$ shows the valence fermion mass dependence of the decay constant in lattice units on several sets of ensembles. The valence fermion mass includes the residual mass determined at each bare valence mass. For $N_{f} \leq 4$, the data points at different dynamical fermion mass $m$ are close to each other, and importantly the decay constant has finite values in the chiral limit, whereas those are split at $N_{f}=6$. At $N_{f}=8$, the decay constant in the $m_{v a l} \rightarrow 0$ limit seems to vanish as the dynamical fermion mass decreases.

Since the lattice scale significantly depends on $m$ for larger values of $N_{f}$, we show, in Figure $\square$, the same plot, but the both axes are scaled with the measured $r_{0}$ (shown in Table $\mathbb{W}$ ). We confirm that the above tendency is unchanged. These results are consistent with the observation in the study of the meson spectrum [ [5] that the chiral symmetry in the vacuum is broken at $N_{f}=2$ and 4 and unbroken at $N_{f}=8$. The $N_{f}=6$ case requires more detailed analysis in the small mass region to draw a conclusive picture. Since the standard domain-wall fermion used in this work has rather large residual mass, we consider various improvements including the link smearing [ [ $]$ ] and/or the optimal domain-wall formulation [Q].

\section{Adjoint fermion}

This section reports our numerical investigation of the $\mathrm{SU}(2)$ gauge theory with adjoint Wilson fermions. We start with a simulation with the topology fixing term [एव, प],

$$
\operatorname{det}\left(\frac{H_{W}^{2}}{H_{W}^{2}+\mu^{2}}\right)=\int \mathscr{D} \chi^{\dagger} \mathscr{D} \chi \exp \left[-S_{E}\right]
$$

$H_{W}=\gamma_{5} D_{W}$ is the Hermitian Wilson Dirac operator with

$$
D_{W}=4-M_{0}-\frac{1}{2} \sum_{\mu}\left[\left(1-\gamma_{\mu}\right) U_{A \mu}(x) \delta_{x+\hat{\mu}, y}+\left(1+\gamma_{\mu}\right) U_{A \mu}^{\dagger}(x-\hat{\mu}) \delta_{x-\hat{\mu}, y}\right]
$$



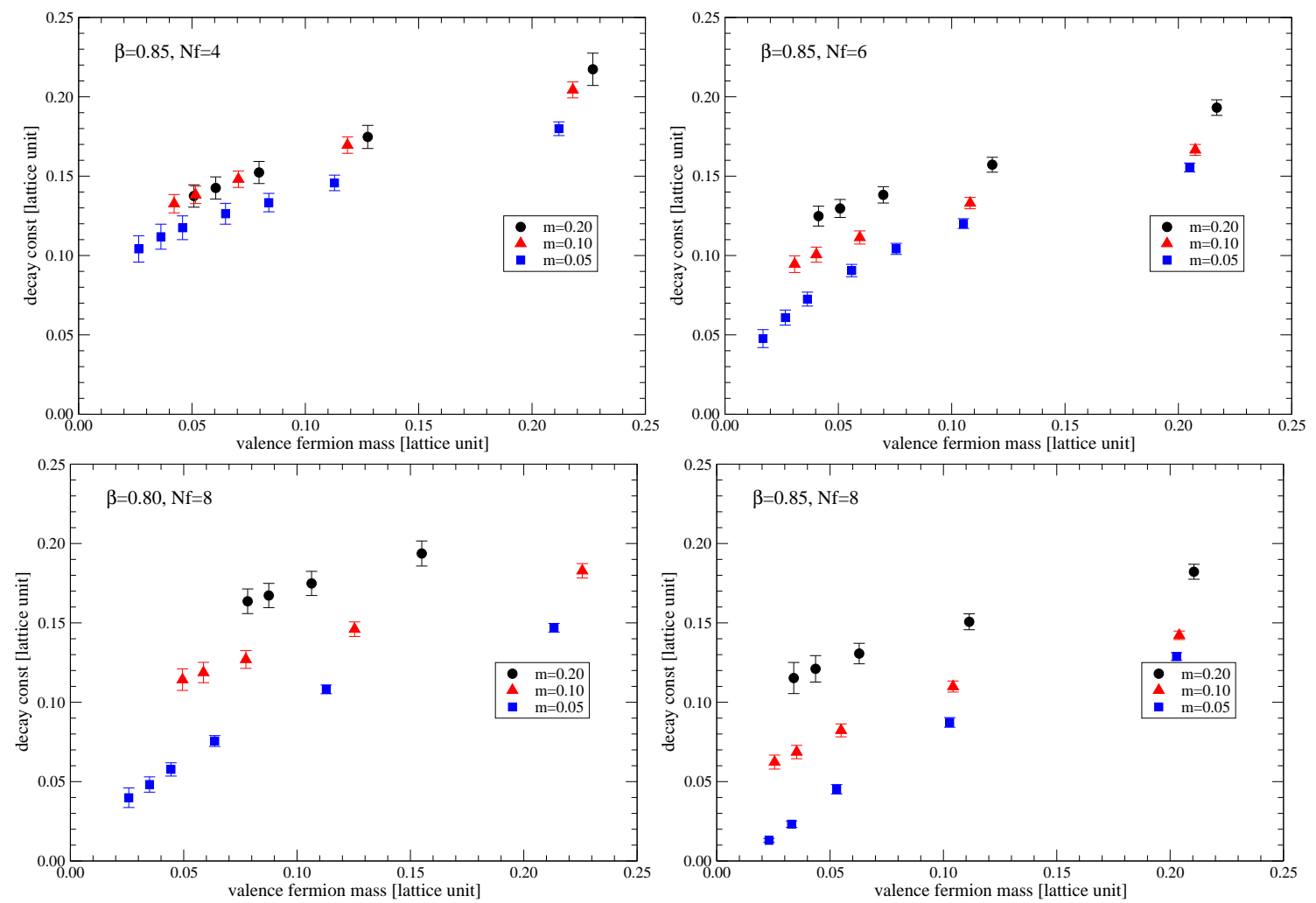

Figure 1: The valence fermion mass dependence of the pseudoscalar decay constant on four different ensembles with the fundamental domain-wall fermions.

where $\left(U_{A}\right)^{a b}=2 \operatorname{tr}\left[T_{F}^{a} U T_{F}^{b} U^{\dagger}\right], T_{F}$ the generator of the fundamental representation. The action (B.D) suppresses near-zero modes of $H_{W}$ and hence the topological charge is kept unchanged. For the gauge action, the Iwasaki action is employed. With the topology fixing term, we had expected that in between the fingers of Aoki phase, the system resembles the quenched one, namely it is confined and chiral symmetry broken.

Figure [3] shows the result on a $8^{3} \times 16$ lattice at $\beta=0.90$ and $M_{0}$ in the range 1.4-2.0 [ए0]]. The value of the twisted ghost mass is set to $\mu=0.2$. The left panel shows the hadronic radius $r_{0}$ obtained from the static potential in the fundamental representation. For $M_{0}<1.51, r_{0}$ is relatively small, which indicates that the system is in the confined phase and the two-flavors of Wilson fermions in the topology fixing term describe physical fermions. The critical $M_{0}$ seems to lie between 1.51 and 1.52. At $M_{0}=1.52$, the static potential is well described by a combination of the Coulomb potential and a constant term (i.e. without the linear potential). For $M_{0}>1.52$, while the linear term has non-vanishing contribution, the values of $r_{0}$ is quite large. These results suggest that the confining nature is much restrained compared to the physical side of $M_{0}$, i.e. $M_{0}<1.51$.

Since in the form of Eq. (B.J), the degrees of the cancellation of high frequency modes in the numerator and the denominator is controlled by the twisted ghost mass $\mu$, we study the $\mu$ dependence of $r_{0}$. The right panel of Figure [3 shows the result at $M_{0}=1.8$. As $\mu$ decreases, $r_{0}$ decreases as well so that the confining feature becomes manifest. This result indicates that, in order for the topology fixing term not to destroy the features of the original vacuum, the twisted ghost 

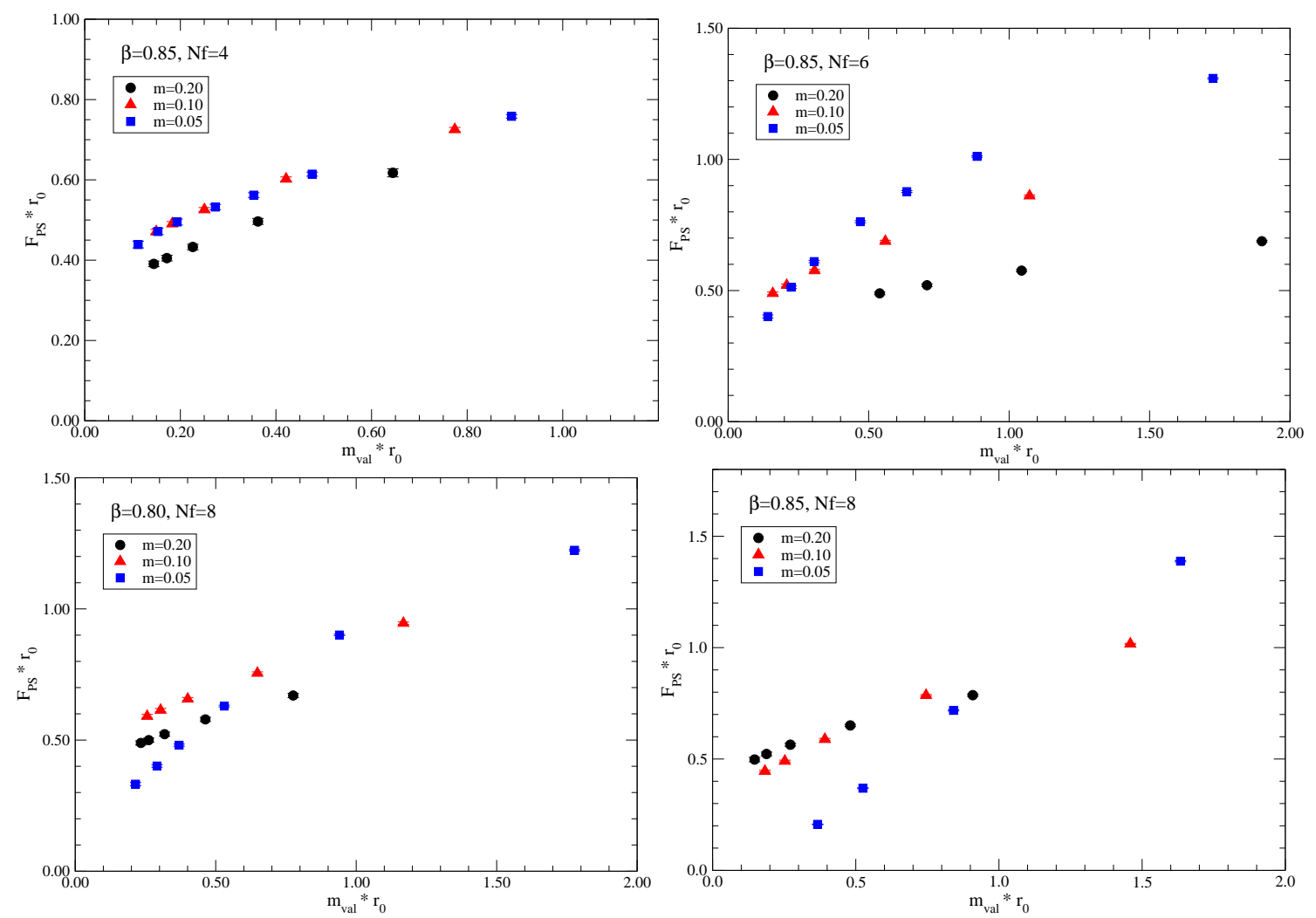

Figure 2: The same results as Fig. $⿴$ but in the $r_{0}$ unit.
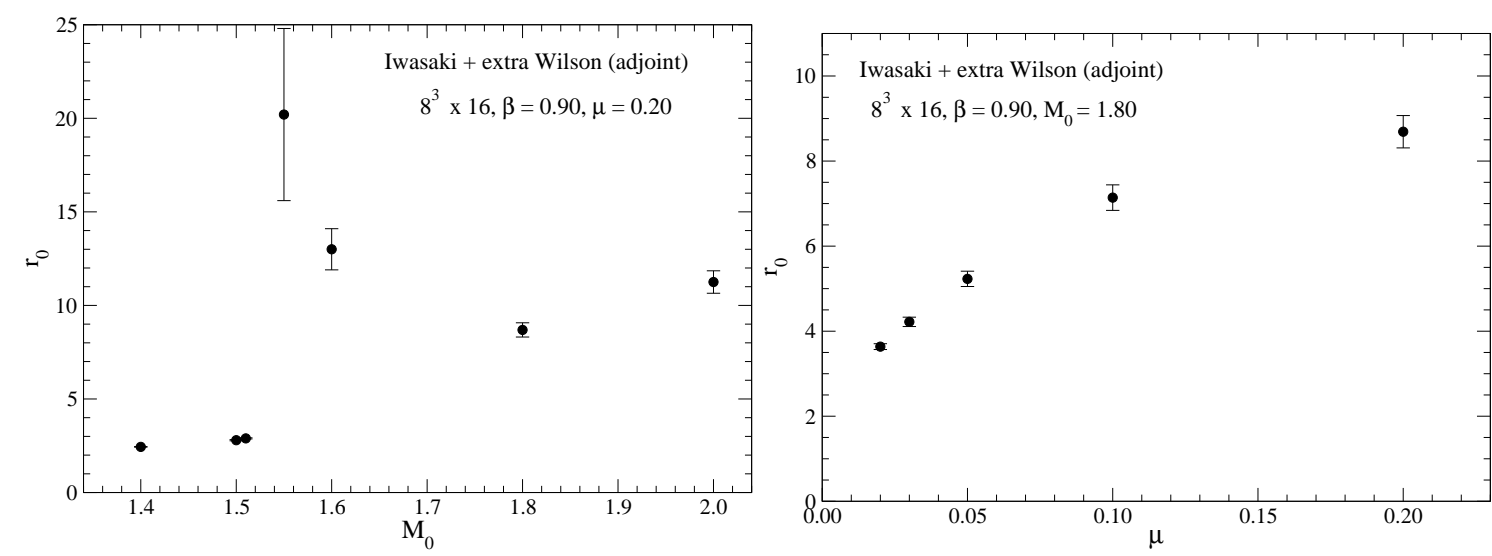

Figure 3: The hadronic radius, $r_{0}$, obtained on $8^{3} \times 16$ at $\beta=0.90$. The $M_{0}$ dependence at the twisted mass $\mu=0.2$ (left) and $\mu$ dependence at $M_{0}=1.8$ (right) are shown.

mass parameter has to be sufficiently small. This is a distinct feature of the adjoint fermion case where at $N_{f}=2$ the nature of the system is sensitive to the low-lying modes of the Wilson-Dirac operator. On the other hand, at the large $\mu$ region, $r_{0}$ is as large as the current lattice size so that the confining becomes less clear due to the finite size effect. This is in contrast to the fundamental case where the confining would be restored as far as the system is apart from the Aoki phase.

Motivated by the above result for the simulations with the topology fixing term, we start a study 

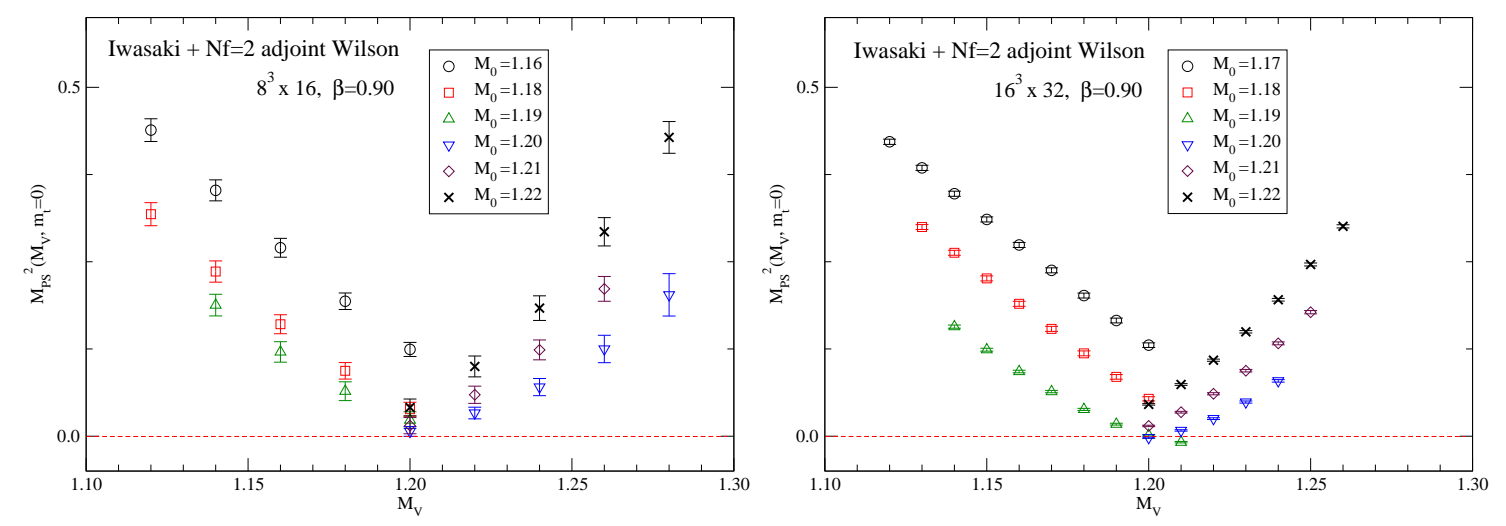

Figure 4: Search for the onset of the Aoki phase in $N_{f}=2$ adjoint Wilson fermions.

of the Aoki phase of the dynamical adjoint Wilson fermions without the ghost fields. We generate configurations with two dynamical flavors of adjoint Wilson fermions, with the same setup as those with the topology fixing term other than the fermion part. To probe the Aoki phase, following Ref. [ㅁ] ], we introduce the twisted mass term as an external field in the Wilson-Dirac fermion action, $D_{W t m}=D_{W}-i m_{t} \tau_{3} \gamma_{5}[\mathbb{W}]$ ]. In the Aoki phase, in the limit of $m_{t} \rightarrow 0, \pi_{3}$ becomes massive while $\pi_{ \pm}$remains massless. Thus we measure the pseudoscalar meson correlator corresponding to $\pi_{ \pm}$for several values of $m_{t}$.

Figure $⿴$ $\rightarrow$ shows the results at $\beta=0.90$ on $8^{3} \times 16$ (left) and $16^{3} \times 32$ (right) lattices. At each value of $M_{0}$, we measure the pseudoscalar meson correlator for several values of valence mass $M_{V}$ and the twisted mass $m_{t}$, and then extrapolate them to $m_{t}=0$. Figure $⿴$ displays that the finite size effect is small and the behavior of $M_{P S}^{2}$ is essentially the same for two lattice sizes. Tracing the points with $M_{V}=M_{0}$, the transition lies around $M_{0}=1.2$, beyond which the meson mass tends to increase. This observation indicates that the Aoki phase is relatively narrow at this $\beta$.

We are performing the calculation at several other values of $\beta$. More detailed analyses in the presence of the topology fixing term or $N_{f}=2$ Wilson fermions are underway, where the eigenvalue spectrum of $H_{W}$ and the topological charge are investigated.

\section{Conclusion and outlook}

In this paper we investigate the $\mathrm{SU}(2)$ gauge theories with fermions in the fundamental and adjoint representations. For the fundamental representation, $N_{f}$-dependence of the meson spectrum and decay constant are examined with the standard domain-wall fermions for $N_{f}=2,4,6$, and 8 at two or three values of $\beta$ and at three values of fermion mass $m$. The results indicate that the chiral symmetry is broken at the massless limit on $N_{f}=2$ and 4 , while unbroken at $N_{f}=8$. For conclusive results for $N_{f}=6$, it is necessary to perform the simulation at smaller mass region which is inaccessible with currently used standard domain-wall fermions. Extension to the improved domain-wall formulation is now underway.

For the adjoint fermions, we investigate the system with two flavors of Wilson fermions with and without the twisted mass ghost. We showed that the nature of the system is sensitive to the twisted mass of the ghost, and determined the critical fermion mass corresponding to the edge of the Aoki phase. Application of the domain-wall or overlap fermions to this system is now 
in progress. One interesting topic with the chirally symmetric fermions is a measurement of the fractional topological charge [[3]. While Ref. [14] argued that the fractional topological charge is lattice artifact and disappears in the continuum limit based on simulation of the quenched SU(3) gauge theory, direct lattice calculations are important.

Numerical simulations were performed on Hitachi SR16000 and IBM Blue Gene/Q at KEK under a support of its Large-scale Simulation Program (Nos.12/13-15, 13/14-14, 14/15-22) and $\varphi$ computer system at KMI, Nagoya University. We also thank the Japan Lattice Data Grid which is a grid file system constructed on a virtual private network SINET4 provided by National Institute of Informatics for efficient data transfer. A part of simulation codes used in this work is based on the Bridge++ code set [[15]. This work is supported in part by the Grand-in-Aid for Scientific Research of Japan (Nos.22224003, 22740183, 25400284, 15H03669).

\section{References}

[1] K. Yamawaki, M. Bando and K. i. Matumoto, Phys. Rev. Lett. 56, 1335 (1986).

[2] For reviews on technicolor model, see, for example, C. T. Hill and E. H. Simmons, Phys. Rept. 381, 235 (2003) [Erratum-ibid. 390, 553 (2004)] [hep-ph/0203079]; F. Sannino, arXiv:0804.0182 [hep-ph].

[3] For recent activities on the lattice, see, for example, J. Kuti, PoS LATTICE 2013, 004 (2014).

[4] H. Matsufuru, Y. Kikukawa, K. i. Nagai and N. Yamada, PoS LATTICE 2013, 123 (2014) [arXiv:1401.6655 [hep-lat]].

[5] H. Matsufuru, K. i. Nagai and N. Yamada, PoS LATTICE 2014, 241 (2014).

[6] P. M. Vranas, hep-lat/0001006; T. Izubuchi et al. [RBC Collaboration], Nucl. Phys. Proc. Suppl. 106, 748 (2002) doi:10.1016/S0920-5632(01)01833-3

[7] H. Fukaya et al. [JLQCD Collaboration], Phys. Rev. D 74, 094505 (2006) doi:10.1103/PhysRevD.74.094505 [hep-lat/0607020].

[8] C. Morningstar and M. J. Peardon, Phys. Rev. D 69, 054501 (2004) doi:10.1103/PhysRevD.69.054501 [hep-lat/0311018].

[9] T. W. Chiu, Phys. Rev. Lett. 90, 071601 (2003) doi:10.1103/PhysRevLett.90.071601 [hep-lat/0209153].

[10] H. Matsufuru, Y. Kikukawa, K. -I. Nagai and N. Yamada, PoS LATTICE 2010, 090 (2010); H. Matsufuru, Y. Kikukawa and N. Yamada, PoS LAT 2009, 064 (2009).

[11] S. W. Kim et al. [JLQCD Collaboration], PoS LATTICE 2011, 069 (2011) [arXiv:1111.2180 [hep-lat]].

[12] M. Golterman, Y. Shamir and B. Svetitsky, Phys. Rev. D 71, 071502 (2005) doi:10.1103/PhysRevD.71.071502 [hep-lat/0407021].

[13] R. G. Edwards, U. M. Heller and R. Narayanan, Phys. Lett. B 438, 96 (1998) doi:10.1016/S0370-2693(98)00951-4 [hep-lat/9806011].

[14] Z. Fodor, K. Holland, J. Kuti, D. Nogradi and C. Schroeder, JHEP 0908, 084 (2009) doi:10.1088/1126-6708/2009/08/084 [arXiv:0905.3586 [hep-lat]].

[15] S. Ueda et al., PoS LATTICE 2013, 412 (2014); J. Phys. Conf. Ser. 523, 012046 (2014). 